\title{
Initial Modified Pulmonary Index Score Predicts Hospital Length of Stay for Asthma Subjects Admitted to the Pediatric Intensive Care Unit
}

\author{
Andrew G Miller, Kaitlyn E Haynes, Rachel M Gates, Kanecia O Zimmerman, \\ Kathleen W Bartlett, Heather S McLean, and Kyle J Rehder
}

\begin{abstract}
BACKGROUND: Scoring systems are frequently used to assess the severity of pediatric asthma exacerbations. The modified pulmonary index score (MPIS) has been found to be highly correlated with length of stay (LOS) in the pediatric intensive care unit (PICU). We sought to evaluate the use of the MPIS to predict hospital LOS for patients admitted to our PICU. METHODS: We retrospectively reviewed the medical records of pediatric asthma subjects aged 2-17 y admitted to our PICU between June 2014 and November 2017. We divided subjects a priori into 3 groups (low: MPIS 0-5; medium: MPIS 6-9; high: MPIS $\geq 10$ ) based upon each subject's first MPIS documented in the PICU. Hospital LOS, PICU LOS, time on continuous albuterol, and increased respiratory support were compared between groups. RESULTS: 143 subjects were included. There were no differences for demographics, medical history, cause of exacerbations, or mean heart rate between groups. There were significant differences between groups for mean breathing frequency $(P<.001), \mathrm{S}_{\mathrm{pO}_{2}}(P=.01)$, and $\mathrm{F}_{\mathrm{IO}_{2}}(P<.004)$. There were significant differences between groups for route of admission $(P=.02)$, high-flow nasal cannula use $(P<.001)$, and use of a helium-oxygen mixture $(P<.001)$. There were significant differences between groups for median hospital $\operatorname{LOS}(1.2$ vs 2.3 vs $3.4 \mathrm{~d}, P<.001)$, PICU LOS (0.39 vs 1.3 vs $2 \mathrm{~d}, P<.001)$, and time on continuous albuterol $(7.4$ vs 20.6 vs $34.7 \mathrm{~h}$, $P<.001)$. After adjusting for demographics and medical history, the incidence risk ratio for hospital LOS was 2.09 for PICU admission for an MPIS of 6-9 and 2.68 for an MPIS $\geq 10$ when compared to an MPIS < 6. CONCLUSIONS: The MPIS thresholds used in our pathway appropriately predicted LOS in our cohort of subjects with asthma admitted to the PICU. Higher MPIS was associated with increased hospital LOS, PICU LOS, and time on continuous albuterol. Key words: asthma; pediatric; children; asthma scoring; MPIS; modified pulmonary index score; status asthmaticus; pediatric intensive care unit. [Respir Care 2020;65(9):1227-1232. (C) 2020 Daedalus Enterprises]
\end{abstract}

\section{Introduction}

Status asthmaticus is a common reason for admission to the pediatric intensive care unit (PICU). ${ }^{1}$ Objective determinations of illness severity in pediatric patients with asthma are

\footnotetext{
Mr Miller, Ms Haynes, and Ms Gates are affiliated with Respiratory Care Services, Duke University Medical Center, Durham, North Carolina. Drs Zimmerman and Rehder are affiliated with the Division of Pediatric Critical Care Medicine, Duke University Medical Center, Durham, North Carolina. Drs Bartlett and McLean are affiliated with the Division of Pediatric Hospital Medicine, Duke University Medical Center, Durham, North Carolina.

Ms Gates presented a version of this paper as an Editors' Choice abstract at AARC Congress 2019, held November 9-12, 2019, in New Orleans, Louisiana.
}

challenging because smaller children and those in respiratory distress are unable to provide reliable bedside spirometry or peak flow measurements. ${ }^{2}$ Thus, alternatives to objective measures of lung function are needed. Asthma scoring systems are

\footnotetext{
Mr Miller has disclosed a relationship with Ventec Life Systems. The other authors have disclosed no conflicts of interest.

Supplementary material related to this paper is available at http://www. rcjournal.com.

Correspondence: Andrew G Miller MSc RRT RRT-ACCS RRT-NPS, Duke University Medical Center, 2301 Erwin Rd, Durham, NC 27710. E-mail: andrew.g.miller@duke.edu.
}

DOI: $10.4187 /$ respcare.07396 
used to evaluate illness severity in pediatric patients with asthma and are frequently incorporated into asthma pathways, including those in place in our institution. ${ }^{3-8}$

\section{See the Related Editorial on Page 1413}

We incorporated the modified pulmonary index score (MPIS) into our pediatric asthma protocol because it has been shown to have a high interobserver correlation between respiratory therapists, nurses, and physicians. ${ }^{4}$ The MPIS contains 3 objective parameters and 3 subjective parameters. Each parameter is scored from 0 to 3, with a total index score ranging from 0 to 18 . (Table 1 ). This correlation is supported by several studies. ${ }^{5,6}$ In addition to a high interobserver correlation, a higher MPIS has also been reported to be correlated with length of stay (LOS) in the PICU ${ }^{4,5}$ and the need for hospital admission in subjects evaluated in the emergency department. ${ }^{9}$ These studies have been relatively small and did not manage asthma using a clinical pathway. Thus, we sought to evaluate the use of the MPIS to predict hospital LOS for patients admitted to our PICU and managed using a standardized clinical pathway.

\section{Methods}

Following institutional review board approval, the medical records of all pediatric patients with asthma who were 2-17 y old and admitted to our PICU between June 2014 and November 2017 (after implementation of our pediatric asthma protocol) were retrospectively reviewed. Subjects were identified via International Classification of Diseases, 9th Revision and 10th Revision codes and were managed via our pediatric asthma protocol, which has been described in detail elsewhere. ${ }^{3,7}$ In brief, bronchodilator frequency is managed by respiratory therapists using a standardized protocol and can be increased or decreased independently based on the MPIS. Helium-oxygen mixture (heliox) and intravenous magnesium are given if the MPIS is $\geq 12$. All our PICU patients initially receive systemic corticosteroids every $6 \mathrm{~h}$. Noninvasive ventilation and high-flow nasal cannula (HFNC) may be utilized but are not prescribed as part of the pathway. As part of clinical practice, HFNC prongs were sized to half the diameter of the patient's nares, and initial flow was titrated to meet the patient's inspiratory flow demand and $\mathrm{S}_{\mathrm{pO}_{2}}$ goal. $\mathrm{F}_{\mathrm{IO}_{2}}$ was titrated to keep $\mathrm{S}_{\mathrm{pO}_{2}}>92 \%$ as part of our asthma protocol. Adjustments to the HFNC flow was determined by the care team. We did not record the flows used. Patients receiving continuous albuterol for $>4 \mathrm{~h}$ were required to be in our PICU or stepdown unit during the study. Our PICU is staffed around the clock by pediatric intensivists, critical care fellows, pediatric nurse practitioners, and dedicated pediatric respiratory therapists.

\section{QUICK LOOK}

\section{Current knowledge}

Objective determinations of illness severity in pediatric patients with asthma are challenging because smaller children and those in respiratory distress are unable to provide reliable bedside spirometry or peak flow measurements. Asthma scoring systems are used to evaluate illness severity in pediatric patients with asthma and are frequently incorporated into asthma pathways.

\section{What this paper contributes to our knowledge}

The initial modified pulmonary index score (MPIS) was predictive of illness severity and trajectory in pediatric subjects with status asthmaticus. A higher MPIS was significantly associated with hospital and pediatric ICU length of stay, as well as time on continuous albuterol. Subjects with a higher MPIS were more likely to receive support in the form of a helium-oxygen mixture or high-flow nasal cannula, decisions that were independent of the asthma pathway. Total MPIS was the only factor noted in multivariable analysis to be associated with length of stay.

Data were collected on subject age, weight, gender, home medications, medical history, cause of exacerbation, route of admission, respiratory support during admission, PICU readmission, vital signs, MPIS, time on continuous albuterol, and LOS. Routes of admission included transfers from outside hospitals, the emergency department, and inpatient wards. We divided subjects a priori into 3 groups based upon each subject's first MPIS documented in PICU, categorized as low (MPIS 0-5), medium (MPIS 6-9), and high (MPIS $\geq 10$ ). We chose these groups based on the thresholds used for escalation and de-escalation in our asthma pathway These groupings are similar to those reported in other studies of MPIS. ${ }^{5,10}$ A second analysis was performed by dividing MPIS into 5 a priori categories $(<6,7-8,8-9,10-11$, and $\geq 12)$ because we observed clinically that a difference of 2 points was clinically important. Hospital LOS, PICU LOS, time on continuous albuterol, and need for increased respiratory support were compared between groups. Data were analyzed with SPSS 24 (IBM, Armonk, New York) with nonparametric, chi-square, and one-way analysis of variance performed for non-normally distributed data, categorical variables, and normally distributed continuous variables, respectively. Because LOS data were nonnormally distributed and positively skewed, Poisson regression analysis was performed to identify PICU admission factors associated with hospital LOS. Statistical significance was set at $\alpha<0.05$. 
Table 1. Components of the Modified Pulmonary Index Score

\begin{tabular}{|c|c|c|c|c|}
\hline MPIS groups & 0 & 1 & 2 & 3 \\
\hline $\mathrm{S}_{\mathrm{pO}_{2}}, \%$ & $>95$ & $93-95$ & 90-92 & $<90$ \\
\hline Accessory muscle use & None & Mild & Moderate & Severe \\
\hline Inspiratory/expiratory ratio & $2: 1$ & $1: 1$ & $1: 2$ & $1: 3$ \\
\hline Wheezing & None & End-expiratory & $\begin{array}{l}\text { Inspiratory/expiratory } \\
\text { wheezing, good aeration }\end{array}$ & $\begin{array}{l}\text { Inspiratory/expiratory } \\
\text { wheezing, decreased aeration }\end{array}$ \\
\hline \multicolumn{5}{|l|}{ Heart rate, beats/min } \\
\hline$<3$ y old & $<120$ & $120-140$ & $141-160$ & $>160$ \\
\hline$\geq 3$ y old & $<100$ & $100-120$ & $121-140$ & $>140$ \\
\hline \multicolumn{5}{|c|}{ Breathing frequency, breaths/min } \\
\hline$<6$ y old & $\leq 30$ & $31-45$ & $46-60$ & $>60$ \\
\hline$\geq 6$ y old & $\leq 20$ & $21-35$ & $36-50$ & $>50$ \\
\hline
\end{tabular}

\section{Results}

A total of 143 subjects were included. There were no differences between groups for age, gender, weight, home medications, medical history, cause of exacerbations, noninvasive ventilation use, or need for intubation. Two subjects in the low MPIS group had bi-level positive airway pressure ventilation (BPAP) started in the emergency department and were weaned from BPAP support in the PICU. Both subjects spent $<24 \mathrm{~h}$ in the PICU. There were significant differences between groups for route of admission $(P=.02)$, HFNC use $(P<.001)$, and heliox use $(P<$ .001 ) (Table 2 ). There was only 1 PICU readmission during the study period.

Significant differences were noted between 3 MPIS groups for mean respiratory frequency $(P<.001), \mathrm{S}_{\mathrm{pO}_{2}}(P=$ $.01)$ and $\mathrm{F}_{\mathrm{IO}_{2}}(P=.004)$, but not respiratory frequency $(P=$ .22). There were also significant differences between groups for median hospital $\operatorname{LOS}(1.2$ vs 2.3 vs $3.4 \mathrm{~d}, P<.001)$, PICU LOS ( 0.39 vs 1.3 vs $2 \mathrm{~d}, P<.001)$, and time on continuous albuterol (7.4 vs 20.6 vs 34.7 h, $P<.001$ ). (Table 3; Figs. 1 and 2). After adjusting for demographics and medical history, the incidence risk ratio for hospital LOS was 2.09 (95\% CI 1.39-3.12, $P<.001$ ) for PICU admission MPIS $6-$ 9 and 2.68 (95\% CI 1.79-4.03, $P<.001)$ for an MPIS $\geq 10$ when compared to an MPIS $<6$ (Table 4).

For the 5 MPIS groups, there were no statistically significant differences for age, weight, home medications, medical history, cause of exacerbation, route of admission, need for intubation, PICU readmission, admission $\mathrm{S}_{\mathrm{pO}_{2}}$, or BPAP use. There were significant differences between groups for hospital LOS $(P<.001)$, PICU LOS $(P<.001)$, time on continuous albuterol $(P=.001)$, respiratory frequency $(P=.02)$, heart rate $(P=.004), \mathrm{F}_{\mathrm{IO}_{2}}(P<.001)$, HFNC use $(P<.001)$, and heliox use $(P=.001)$ (see the supplementary materials at http://www.rcjournal.com).

\section{Discussion}

In our cohort, the initial MPIS was predictive of illness severity and trajectory in pediatric subjects with status asthmaticus. As expected, there were significant differences in respiratory frequency, $\mathrm{S}_{\mathrm{pO}_{2}}$, and $\mathrm{F}_{\mathrm{IO}_{2}}$ between groups because these data are objective components of the MPIS. Thus, it is important confirmation of the validity of the MPIS that hospital LOS, PICU LOS, and time on continuous albuterol were significantly different between groups. There was only 1 PICU readmission during the study period, suggesting that subjects weaned per MPIS protocol were truly ready for de-escalation of care. In addition, subjects with a higher MPIS were more likely to receive heliox or HFNC support, decisions that were independent of the asthma pathway. Total MPIS was the only factor noted in multivariable analysis to be associated with LOS.

In contrast to the original study published by Carroll et $\mathrm{al},{ }^{4}$ which was designed primarily to assess the reproducibility of the scale and evaluate differences in MPIS for those requiring PICU admission, we examined the association of MPIS with subject outcomes. Our study also had a larger sample size and standardized asthma management. ${ }^{4}$ Importantly, the introduction of our asthma protocol that uses the MPIS was associated with decreased hospital LOS for all pediatric subjects with asthma ${ }^{3}$ as well as those in the PICU with status asthmaticus. ${ }^{7}$

Several other studies have used the MPIS to evaluate illness severity in pediatric asthma. Maekawa et $\mathrm{al}^{6}$ evaluated the MPIS in a cohort of 25 children and found a high interobserver agreement; the investigators concluded that the MPIS was a reliable tool to assess asthma severity in children. Their study was designed to evaluate the reproducibly of the MPIS in children, particularly preschool age children in whom evaluating asthma severity is a major challenge. ${ }^{6}$ Maekawa et $\mathrm{al}^{6}$ also evaluated the ability of the MPIS to 
Modified Pulmonary Index Score in Pediatric Asthma

Table 2. Subject Demographics

\begin{tabular}{|c|c|c|c|c|}
\hline MPIS groups & Low $(0-5)$ & Medium (6-9) & High $\geq 10$ & $P$ \\
\hline Subjects & $18(13)$ & $59(41)$ & $66(46)$ & \\
\hline Age, y & $8(3-10)$ & $6(4-10)$ & $7(5-9)$ & .77 \\
\hline Female & $6(33)$ & $26(44)$ & $29(44)$ & .69 \\
\hline Weight, $\mathrm{kg}$ & $31(16.6-39.1)$ & $21.7(16.6-37.9)$ & $24.6(18.8-33.9)$ & .60 \\
\hline \multicolumn{5}{|l|}{ Home medications } \\
\hline Short-acting $\beta_{2}$ agonist & $16(89)$ & $49(83)$ & $56(85)$ & .83 \\
\hline Inhaled corticosteroid & $4(22)$ & $26(44)$ & $26(39)$ & .25 \\
\hline Long-acting $\beta_{2}$ agonist + inhaled corticosteroid & $4(22)$ & $9(15)$ & $11(17)$ & .79 \\
\hline None & $2(11)$ & $9(15)$ & $9(14)$ & .90 \\
\hline \multicolumn{5}{|l|}{ History } \\
\hline Intubation & $1(6)$ & $1(2)$ & $2(3)$ & .68 \\
\hline ICU admission & $2(11)$ & $13(22)$ & $22(33)$ & .11 \\
\hline Noninvasive ventilation & $2(11)$ & $1(2)$ & $3(5)$ & .21 \\
\hline None & $16(89)$ & $46(78)$ & $43(65)$ & .08 \\
\hline Cause of exacerbation & & & & .31 \\
\hline Viral & $9(50)$ & $38(64)$ & $41(62)$ & \\
\hline Unknown & $6(33)$ & $10(17)$ & $16(24)$ & \\
\hline Environmental & $1(6)$ & $3(5)$ & $3(5)$ & \\
\hline Exposure & $0(0)$ & $2(3)$ & $3(5)$ & \\
\hline Nonadherence & $1(6)$ & $6(10)$ & $3(5)$ & \\
\hline Other & $1(6)$ & $0(0)$ & $0(0)$ & \\
\hline \multicolumn{5}{|l|}{ Route of admission } \\
\hline Emergency department & $8(44)$ & $29(49)$ & $26(39)$ & .02 \\
\hline Outside hospital & $9(50)$ & $29(49)$ & $25(38)$ & \\
\hline Stepdown & $0(0)$ & $1(2)$ & $3(5)$ & \\
\hline Hospital wards & $0(0)$ & $0(0)$ & $10(15)$ & \\
\hline Missing & $1(6)$ & $0(0)$ & $2(3)$ & \\
\hline \multicolumn{5}{|l|}{ Respiratory support } \\
\hline High-flow nasal cannula & $4(22)$ & $9(15)$ & $34(52)$ & $<.001$ \\
\hline Noninvasive ventilation & $2(11)$ & $5(8)$ & $8(12)$ & .80 \\
\hline Helium-oxygen mixture & $0(0)$ & $11(19)$ & $32(48)$ & $<.001$ \\
\hline \multicolumn{5}{|l|}{ Admission data } \\
\hline MPIS & $4(3-5)$ & $8(7-8)$ & $12(11-13)$ & $<.001$ \\
\hline Heart rate, beats/min & $136 \pm 21$ & $151 \pm 18$ & $157 \pm 16$ & .22 \\
\hline Breathing frequency, breath/min & $32 \pm 13$ & $37 \pm 11$ & $43 \pm 12$ & $<.001$ \\
\hline $\mathrm{S}_{\mathrm{pO}_{2}}$ & $97 \pm 2.6$ & $96 \pm 2.7$ & $95 \pm 3.3$ & .01 \\
\hline $\mathrm{F}_{\mathrm{IO}_{2}}$ & $0.38 \pm 0.30$ & $0.33 \pm 0.23$ & $0.49 \pm 0.28$ & .004 \\
\hline
\end{tabular}

predict hospital admission in a large cohort of pediatric subjects with asthma. They found that the MPIS showed excellent overlap with Japanese Asthma Guidelines classifications, with the mild group having an MPIS 0-5, the moderate group 6-10, the severe group 11-15, and those with respiratory failure an MPIS $\geq 16 .{ }^{10}$ They did not examine the relationship with admission MPIS and LOS. Buyuktiryaki et $\mathrm{al}^{9}$ used the MPIS to evaluate asthma severity in children presenting to the emergency department in Turkey and found that those hospitalized had a higher MPIS at presentation, at $1 \mathrm{~h}$ after treatment, and at $4 \mathrm{~h}$ after treatment. Multivariable analysis revealed that the MPIS at presentation and at $1 \mathrm{~h}$ were associated with hospital admission. The median MPIS noted in their study in the hospitalized group was 6; thus, it is difficult to compare our results as our illness severity was much higher. Admission criteria likely varied between our centers.

Koga et $\mathrm{al}^{5}$ evaluated the MPIS in a cohort of 87 children with asthma and found the MPIS was highly correlated among different raters. They found an MPIS of 10 was the optimum cutoff for the initiation of continuous bronchodilator therapy, identical to the cutoff for escalation in our protocol. $^{7}$ There was some disagreement in their study between the subjective and objective exacerbation severity. In addition, their study found a moderate correlation between the MPIS and LOS; however, their mean LOS was 


\section{Modified Pulmonary Index Score in Pediatric Asthma}

Table 3. Outcome Data by MPIS Group

\begin{tabular}{|c|c|c|c|c|}
\hline & Low $(0-5)$ & Medium (6-9) & High $\geq 10$ & $P$ \\
\hline Intubated & $0(0)$ & $0(0)$ & $1(2)$ & .47 \\
\hline PICU readmission & $1(6)$ & $0(0)$ & $0(0)$ & .034 \\
\hline Hospital LOS, d & $1.2(1.0-1.5)$ & $2.3(1.5-3.1)$ & $3.4(2.6-4.5)$ & $<.001$ \\
\hline PICU LOS, d & $0.39(0.30-0.72)$ & $1.3(0.63-1.8)$ & $2.0(1.6-3.0)$ & $<.001$ \\
\hline Time on continuous albuterol, $\mathrm{h}^{*}$ & $7.4(0.4-12)$ & $20.6(9.1-35.3)$ & $34.7(21.3-53.3)$ & $<.001$ \\
\hline
\end{tabular}

$\overline{\text { Data are presented as } n}(\%)$ or median (interquartile range).

*For low MPIS: $n=8$ subjects; for medium MPIS: $n=47$ subjects; for high MPIS: $n=63$ subjects.

MPIS $=$ modified pulmonary index score

$\mathrm{PICU}=$ pediatric ICU

LOS $=$ length of stay

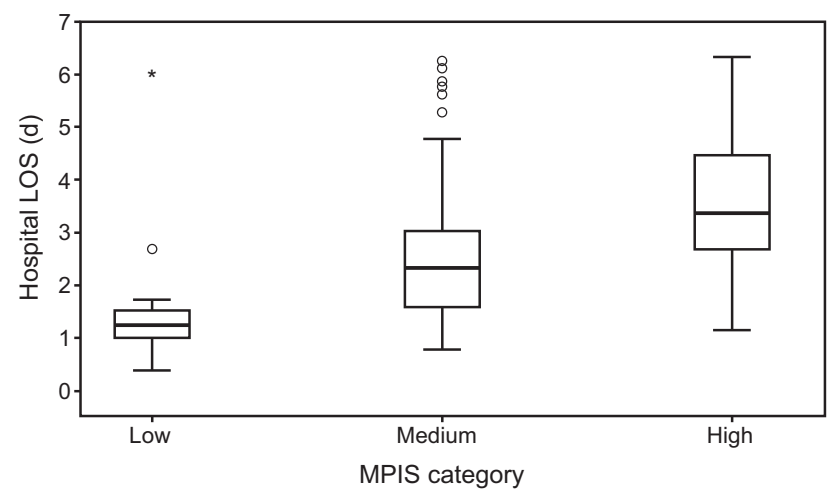

Fig. 1. Box plot of MPIS category for hospital LOS. Low $<6$, Medium $=6-9$, High $\geq 10$. LOS $=$ length of stay; MPIS $=$ modified pulmonary index score.

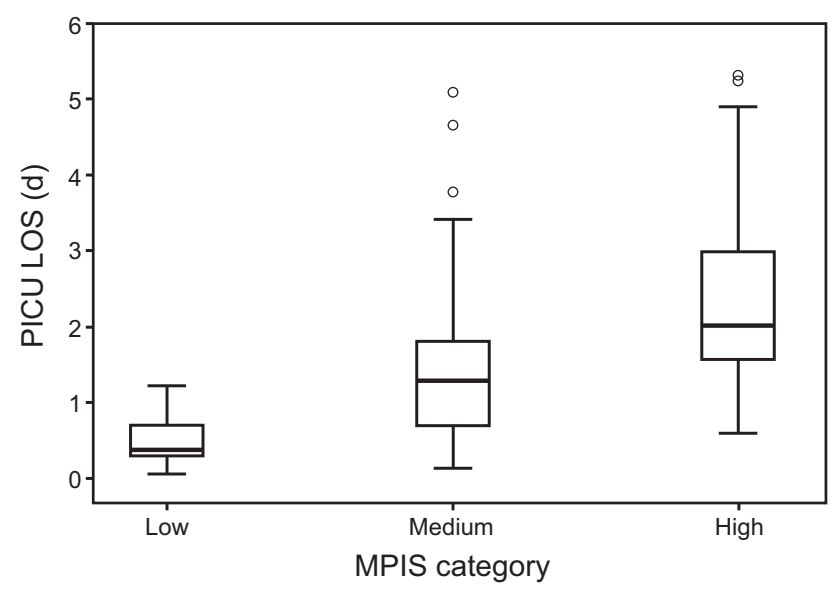

Fig. 2. Box plot of MPIS category for PICU LOS. Low $<6$, Medium $=6-9$, High $\geq 10$. PICU = pediatric ICU; LOS = length of stay; MPIS = modified pulmonary index score.

$6.3 \pm 0.4 \mathrm{~d}$, which was longer than the $3.4 \mathrm{~d}$ reported in our study, probably due to differences in in-patient asthma management between centers. Importantly, they did not evaluate the lower cutoff to identify eligibility for decreasing bronchodilator frequency, their asthma management was not standardized, and they did not evaluate differences in LOS between different groups. We did not specifically evaluate the statistical correlation coefficients between hospital LOS and MPIS at admission; however multivariate analysis revealed MPIS was the only factor that was statistically significantly associated with LOS.

The MPIS differentiates illness severity in pediatric patients with asthma well. Importantly, our study found that an increasing MPIS was associated with an increase in hospital LOS. Although decisions about weaning bronchodilators and discharge readiness in our pathway are based on the MPIS, providers always have the option of deviating from the pathway if a patient is not progressing. Decisions to admit the patient to the PICU from the in-patient wards is driven by the MPIS; at the time of the study, however, the emergency department was not using the MPIS to determine patient disposition. Its ease of use, high interobserver reliability, and robustness in a variety of settings indicate that the MPIS is an excellent tool to evaluate pediatric asthma severity.

\section{Limitations}

Our study has several limitations. As a retrospective review, we were limited to data available in the electronic medical record. The decision to initiate noninvasive ventilation or HFNC was not controlled as part of our asthma pathway. PICU LOS is affected by bed availability on the wards, and there were no standard criteria for PICU discharge. Finally, the use of the MPIS as part of our pathway may have created a self-fulfilling bias because subjects with a higher MPIS may have been treated more aggressively. However, our data demonstrated a clear correlation between the initial MPIS and clinical outcomes, whereas the treatment pathway is guided by the most recent MPIS and should not be biased by prior scoring.

\section{Conclusions}

The MPIS accurately predicted stay and intensity of therapy in our cohort of asthma subjects admitted to the PICU, 


\section{Modified Pulmonary Index Score in Pediatric Asthma}

Table 4. Poisson Regression

\begin{tabular}{lcc}
\hline \hline \multicolumn{1}{c}{ Variable } & $\begin{array}{c}\text { Incidence Rate Ratio } \\
(95 \% \text { CI })\end{array}$ & $P$ \\
\hline MPIS $<6$ & Reference & Reference \\
MPIS 6-9 & $2.09(1.39-3.12)$ & $<.001$ \\
MPIS $>10$ & $2.68(1.79-4.03)$ & $<.001$ \\
Male & $1.06(0.85-1.32)$ & .63 \\
Weight, kg & $1.00(1.00-1.01)$ & .33 \\
Age, y & $1.00(0.95-1.05)$ & .91 \\
Admission route & Reference & Reference \\
$\quad$ Emergency department & $0.86(0.68-1.08)$ & .19 \\
$\quad$ Outside hospital & $0.79(0.47-1.34)$ & .39 \\
$\quad$ Wards & & \\
Cause of exacerbation & Reference & Reference \\
$\quad$ Unknown/other/missing/ & & .30 \\
$\quad$ nonadherence & $1.26(0.82-1.94)$ & .71 \\
$\quad$ Viral & $1.11(0.64-1.94)$ & \\
$\quad$ Environmental/exposure & & .49 \\
Home medications & & .93 \\
Inhaled corticosteroid* & $1.09(0.85-1.39)$ & \\
Long-acting $\beta_{2}$ agonist + & $0.98(0.70-1.39)$ & \\
$\quad$ inhaled corticosteroid & & \\
History of ICU admission & $0.94(0.74-1.21)$ & \\
$\quad$ *Reference, no inhaled corticosteroid & & \\
$\dagger$ Reference, no long-acting $\beta_{2}$ agonist & & \\
MPIS = modified pulmonary index score & & \\
\hline
\end{tabular}

and initial MPIS may be used as a prognostic factor to help triage patients. Higher MPIS was associated with increased hospital LOS, PICU LOS, time on continuous albuterol, and need for HFNC or heliox.

\section{REFERENCES}

1. Hartman ME, Linde-Zwirble WT, Angus DC, Watson RS. Trends in admissions for pediatric status asthmaticus in New Jersey over a 15 year period. Pediatrics 2010;126(4):e904-911.

2. Jones BP, Paul A. Management of acute asthma in the pediatric patient: an evidence-based review. Pediatr Emerg Med Pract 2013;10 (5): $1-23$.

3. Bartlett KW, Parente VM, Morales V, Hauser J, McLean HS. Improving the efficiency of care for pediatric patients hospitalized with asthma. Hosp Pediatr 2017;7(1):31-38.

4. Carroll CL, Sekaran AK, Lerer TJ, Schramm CM. A modified pulmonary index score with predictive value for pediatric asthma exacerbations. Ann Allergy Asthma Immunol 2005;94(3):355-359.

5. Koga T, Tokuyama K, Itano A, Morita E, Ueda Y, Katsunuma T. Usefulness of modified pulmonary index score (mPIS) as a quantitative tool for the evaluation of severe acute exacerbation in asthmatic children. Allergol Int 2015;64(2):139-144.

6. Maekawa T, Oba MS, Katsunuma T, Ishiguro A, Ohya Y, Nakamura H. Modified pulmonary index score was sufficiently reliable to assess the severity of acute asthma exacerbations in children. Allergol Int 2014;63(4):603-607.

7. Miller AG, Haynes KE, Gates RM, Zimmerman KO, Heath TS, Bartlett KW, et al. A respiratory therapist-driven asthma pathway reduced hospital length of stay in the pediatric intensive care unit. Respir Care 2019;64(11):1325-1332.

8. Okada Y, Nakamura T, Maeda M, Ishikawa R, Kamiya T, Imai T. Utility of therapeutic strategy based on the modified Pulmonary Index Score for childhood asthma exacerbation. Allergy Asthma Proc 2019;40(2):111-115.

9. Buyuktiryaki AB, Civelek E, Can D, Orhan F, Aydogan M, Reisli I, et al. Predicting hospitalization in children with acute asthma. J Emerg Med 2013;44(5):919-927.

10. Maekawa T, Ohya Y, Mikami M, Ucmatsu S, Ishiguro A. Clinical utility of the modified pulmonary index score as an objective assessment tool for acute asthma exacerbations in children. JMA J 2018;1(1): 57-66.

This article is approved for Continuing Respiratory Care Education credit. For information and to obtain your CRCE

(free to AARC members) visit

www.rcjournal.com

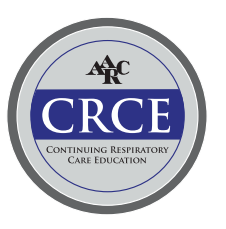

